\title{
EFFECT OF ADDITIONAL DISTANCE MEASUREMENTS ON SATELLITE POSITIONING
}

\author{
Zofia RZEPECKA ${ }^{1)}$, Jacek RAPIŃSKI ${ }^{1) *}$, \\ Slawomir CELLMER ${ }^{1)}$ and Adam CHRZANOWSKI ${ }^{2)}$ \\ ${ }^{1)}$ Institute of Geodesy, University of Warmia and Mazury in Olsztyn, Poland \\ ${ }^{2)}$ Dept. of Geodesy \&Geomatics Engineering, UNB, Fredericton, Canada \\ *Corresponding author's e-mail: jacek.rapinski@gmail.com
}

\begin{tabular}{l} 
ARTiCLE INFO \\
\hline Article history: \\
Received 5 February 2013 \\
Accepted 26 August 2013 \\
Available online 26 November 2013 \\
\hline
\end{tabular}

Keywords:

Geodynamics

Pseudolite (PL)

Kinematic GNSS positioning

Pseudolite-like distance

\begin{abstract}
A prototype of pseudlite (PL), a ground based emitter of GPS signals has been developed at the University of Warmia and Mazury and tested from the hardware point of view. It has also been adapted to work with the Javad Alpha GNSS receiver and with an IFEN SX-NSR software receiver. This paper shows results of studies regarding effects of additional pseudolite-like distances on the accuracy of kinematic satellite positioning. At this stage of the research, only simulated observations with various accuracie shave been used in the analyses. These simulated observations are in the form of distances between PL transmitters and two GNSS receivers forming a baseline.These distances are expressed in cycles and treated as phase measurements. They are double differenced with the reference satellite phase measurements and used along with real observations in a uniform functional model to determine the baseline.

The pseudolites are going to be used in engineering geodetic applications such as deformation monitoring, where often independent positions between the main observational epochs are required.Thus the developed software works in the kinematic mode.

The studies show that the additional observations may help to provide high accuracy of determined positions, but any inaccuracies of these observations affect the results more than similar errors of satellite observations.
\end{abstract}

\section{INTRODUCTION.}

In situations of small number of visible satellites, an augmentation of observables is needed to ensure high positioning accuracy in, for example, kinematic positioning. This kind of positioning is sensitive to changing number of satellites, erroneous observations or DOP factors. Especially, the height component determination can be easily degraded. One possible solution in such cases is to use pseudolites (PL), which are ground-based transmitters of GNSS-like signals. The addition of PLs to the positioning network strengthensthe satellite geometry. In some applications PLs can even replace GNSS satellites.Most often the devices transmit signals of the GPS frequency bands ( $\mathrm{L} 1=1575.42 \mathrm{MHz}$ or/and L2=1227.6 MHz). Both code and carrier phase measurements can be performed. Additional pseudolite-like distances measured to known onground points may help in solving problems of too low accuracy of GNSS positioning caused by small number of satellites or too high DOP values.

In the last period pseudolite equipment has been widely used to a range of applications (Wang, 2002; Wang, 2007), such as aircraft landing (Sherman Lo at faa.gov, Soon et al., 2003; Tsuji et al., 2002), deformation monitoring (Barnes et al., 2002, Barnes et al., 2003; Cosser, 2004; Bond et al., 2007) and even Mars exploration (Lemaster and Rock, 1999). A big step forward in this area was the creation of two systems, developed on the basis of pseudolite technology: Terralites (Novariant, 2005) and Locatalites (Rizos et al., 2011; GPS World, 2011). Locata technology serves for either augmenting GNSS as in the case of poor visible satellites constellation, or to replace GNSS as in indoor applications. Locata is a system of synchronised ground-based transceivers, called LocataLites, which form a network called LocataNet (Rizos et al., 2011). The study aimed to develop their own device that emits pseudo-satellite signals are also conducted by a team of engineering geodesy at the University of Warmia and Mazury in Olsztyn.

A project entitled „Elaboration of Algorithms, Prototype and Geodetic Tests of an On-ground Transmitter of GNSS Signals" is conducted at the Institute of Geodesy, University of Warmia and Mazury UWM in Olsztyn, Poland. Such a transmitting device is called pseudolite (PL). The pseudolitedeveloped at UWM has been constructed on the basis of the Field Programmable Gate Array (FPGA). It enables modification and update of its firmware during the tests and experiments, according to current requirements. It is composed of two parts: digital and analog. The digital part is responsible for pseudorandom code (PRN) and navigation data generation as well asfor user interface (code selection, 
attenuation parameters etc.). This part is clocked by the $16.368 \mathrm{MHz}$ TCXO (Temperature Controlled Cristal Oscillator), which is also used as a reference clock for carrier VCO (Voltage Control Oscilator). The analog part is responsible for carrier generation and its modulation. Hardware basis of the digital part is relied on a DE0 development board from TerraSic, ensuring all essential tools for FPGA. The FPGA is programmed to produce the C/A GPS codes. The programs were written in VHDL language. Before being switched on, the PL is set up to produce one of GPS PRN codes, corresponding to the satellite that is predicted not to be in view during the measurements, or one of the codes reserved for ground transmitters in ICD200D, these are PRNs from 34 to 38. Generation of the navigation message is also performed with the FPGA. Since pseudolite position must be passed to the receivers in different way than that from the satellites, the pseudolite navigation message contains only proper preamble and health bits on every page. Other bits are not used (Pany, 2010).

In analog part of the pseudolite, the carrier frequency (L1) is generated using for this purpose integrated VCO circuit ADF4350 from Analog Devices. The output frequency differs from the nominal L1 frequency $1575.42 \mathrm{MHz}$ by no more than $2 \mathrm{kHz}$, which is about $1.5 \times 10^{-4} \%$. Then, the carrier is modulated, to carry proper information on it. BPSK (Binary Phase Shift Keying) modulation is used like in GPS system. The modulator is based on dedicated integrated circuit RF2638 by RF Micro Devices. Additionally, between the generator and modulator, the attenuator must be used, since maximum acceptable input level for RF2638 is smaller than combined signal carrying $\mathrm{C} / \mathrm{A}$ code and the navigation message (Rapiński et al., 2012).

First test measurements with the pseudolitehave been performed using software receivers SX-NSR (Borre et al., 2007). Currently, observations are taken using Javad Alpha receiver after equipping it with a new firmware. More information on the pseudolite may be found in (Rapiński et al., 2012, Rapiński et al., 2012a). Three more such devices are under construction. Before the real geodetic tests can be performed, software for GNSS positioning augmented with pseudolites must be developed and tested.

General goal of the presented studies is to improve satellite positioning accuracies through application of PL. Before the true PL observations are available, influence of simulated PL-like distances on kinematic determinations is studied. By "kinematic" it is understood here that there is an independent position obtained for each observational epoch and that the positioning algorithm uses phase measurements. There are many possible applications of kinematic methods in geodynamics. It is worth to emphasize that the algorithms developed can be used both in real-time as well as in post-processing systems. They can support detection of motions which are precursors to natural or anthropogenic hazards like landslides (natural or caused by human activity, like e.g.in open pit mines), earthquakes, volcanic activity etc. These phenomena often cause large motions and for the sake of safety it is only needed to verify that the changes of monitored coordinates are not greater than 2-3 cm. Such applications usually need knowledge of coordinate changes in time, like in kinematic methods. To properly use the PL, effects of the additional observations under different conditions, like various number of satellites, DOP factors, geometry of PL location, accuracy of the observation, accuracy of approximate coordinates of the unknown point, should be studied to answer questions such as: when the PL should be incorporated; where should it be placed; what effects are expected;is it worth to use more than one PL; are there any disadvantages or phenomena that need special attention?

Simulationswith additional distances in the form of artificially generated phase measurementsbetween a PL transmitter and two GPS receivers were shortly signaled in (Rapinski et al., 2011). In this paper, observations from three PL transmitters are simulated. In chapter 2 description of the computations and some basic results are given. Also results obtained earlierare shortly referenced. In chapter 3, decrease of mean errors after use of simulated pseudolite observations is treated together within sequential adjustment. In chapter 4 , effect of disturbed simulated observations is studied.

\section{COMPUTATIONAL TESTS.}

Software developed by the authors has been used in the presented studies. It computes 3D positions of the unknown point, relatively to the reference station, independently for each observation epoch. Known ambiguities were admitted. They were computed earlier from first few epochs of observations (Rzepecka, 2004).Standard functional model for double differenced phase observations with known ambiguities was used (Hofmann-Wellenhof et al., 2001). The stochastic model takes into consideration the correlations between the double differenced observations.Approximate position of unknown point were obtained from SPP (Single Point Positions), together with receivers clock correction estimates. Double differenced ionosphere corrections were computed in successive epochs taking advantage of known ambiguities (Dach et al., 2007). Tropospheric corrections were determined using the Saastamoinen model with standard meteorological parameters. More about the software and algorithms may be found in (Rzepecka, 2004). Both the troposphere and ionosphere corrections were applied only to the real GPS observations. The term $\operatorname{Ion}_{A, B}^{r e f, P L}$ was assumed to be equal to zero.

The simulated additional distance observations, expressed in cycles, result in additional double differences used in the adjustment. It was admitted that: 


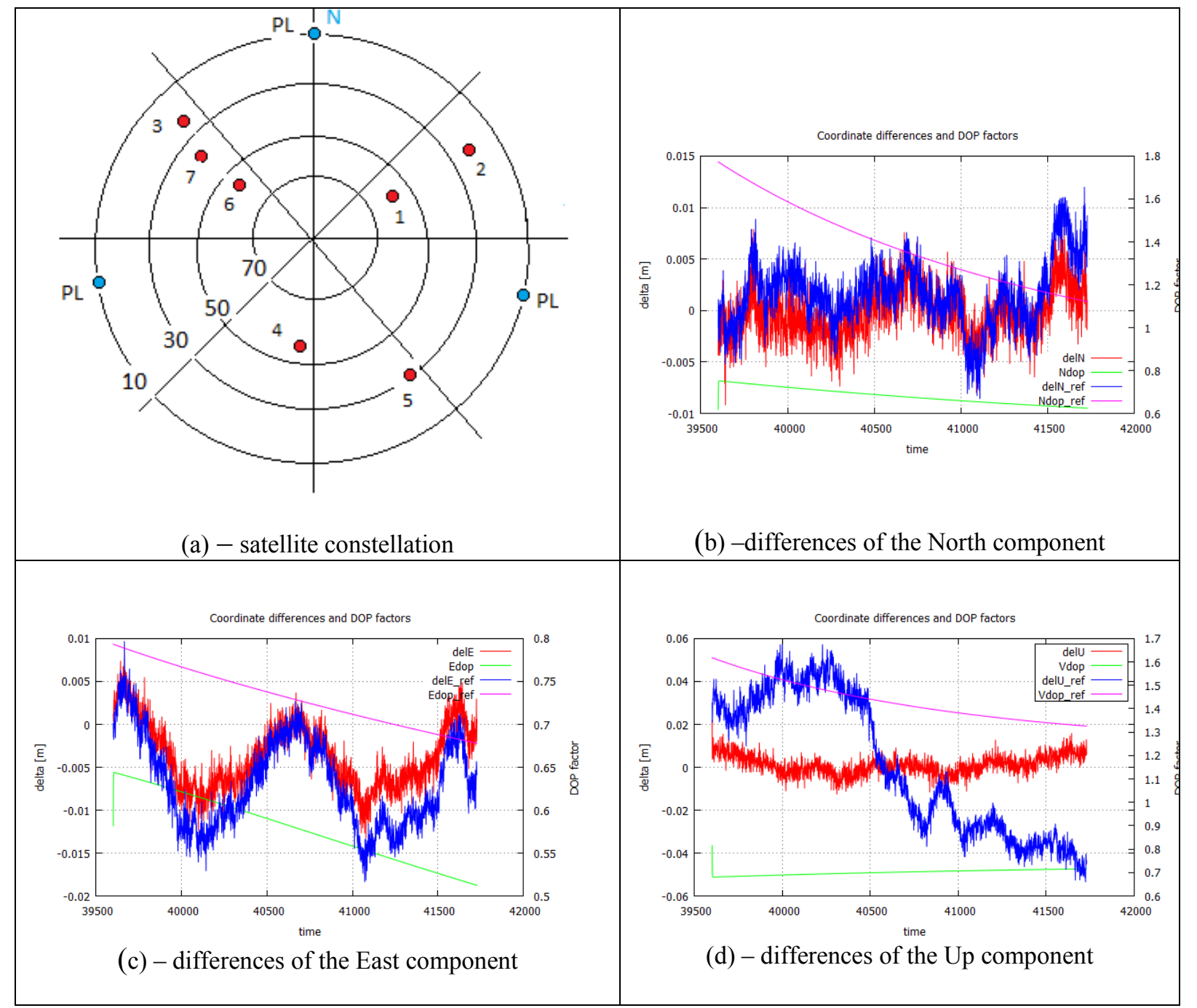

Fig. 1 Comparisons of kinematic solutions to the known positions expressed in local ENU system for the case of 7 satellites and the same 7 satellites complemented with 3 PLs; the solution without PL are referred to as reference ("ref").

$\varphi_{A, B}^{r e f, P L}=\frac{1}{\lambda} \rho_{A, B}^{r e f, P L}$

where:

$\varphi_{A, B}^{r e f, P L}$ - double differenced phase observations for stations $\mathrm{A}, \mathrm{B}$, and transmitters ref (real satellite used as reference) and $\mathrm{PL}$ - the pseudolite simulated

$\rho_{A, B}^{r e f, P L}$ - double differenced geometrical distance, computed on the basis of known coordinates admitted as true.

In all investigations it was assumed that:the additional distances are incorporated into the functional model, the coordinates of the simulated PL location are known and that this additional distance was measured with an accuracy of $2 \mathrm{~mm}$ (it was realized using normally distributed values with standard deviation $\sigma=2 \mathrm{~mm}$ to disturb the simulated observation). It was also assumed here that these distances were measured both at the reference and at the unknown stations. It is important for the studies, since in such a case some errors like not accurate coordinates of the PL, are reduced or totally removed.The distances between the simulated pseudolite and the receivers were equal to some hundreds meters. Since they were similar, the linearization problem has not been taken into regard (Cellmer and Rapinski, 2010).

The software will constitute the basis for common processing of PL together with satellite phase observations, within common least squares procedure. Of course, another adjustment method can be used, but it is important that the functional model comprises both the satellite and pseudolite observations.

In (Rapinski et al., 2011) the previously conducted studies are shortly. The first investigations were performed using only one simulated pseudolite. It was shown that the pseudolite-like distance 
observations improve kinematic results, especially the height component. The influence is larger for poorer satellite constellations. Also it was confirmed that the results are better when the pseudolites are located at such azimuth that their signals come to the receiver from those directions where there are no satellites. It is worth to emphasize that the number of pseudolites used can be larger than one. It was proved during construction of the PL that when electronic parts and integrated circuits are properly chosen, the inner programs are written and tested, than the cost of the device is significantly lower (Rapinski et al., 2012). Thus keeping in mind the encouraging results obtained with one simulated observation, the case of three PLs is studied.

The simulated location of the pseudolite was at the ellipsoidal height 5 meters greater than the height of the unknown point. Since the distance from this point to the PL was admitted as 500 meters, the elevation angle can be computed as only about $0.6^{0}$.

The full satellite constellation used in computations is given in Figure 1a. In Figures $1 \mathrm{~b}$ to 1d., kinematic results compared to the known coordinates are given. In all the plots, the following symbols have been used: delE, delN, delU state for the differences between the known and computed coordinates, expressed in the local East-North-Up system. The kinematic results obtained for the case with full satellite constellation and without any pseudolite are referred to as reference results. Therefore, in the plots, the differences computed for solutions without PL augmentation, are referenced to as delE_ref, delN_ref and delU_ref respectively. In addition, in some plots, the DOP factors are also given. They have been computed as geometrical components of the dilution of precision, consistently for the East, North and Up directions (Strang and Borre, 1997). The symbols used for them are Edop, Ndop, Vdop. When they are used with the extension "_ref" then they refer to the case without the PL. Time units in the plots are given in seconds of day.

In Figure 1, it can be seen that for the reference solution, the differences from the known coordinates fall into the following approximate ranges: $\pm 1 \mathrm{~cm}$ for $\mathrm{N}, \pm 1.5 \mathrm{~cm}$ for $\mathrm{E}$, and $\pm 6 \mathrm{~cm}$ for Up coordinates. After adding three additional distances measured to simulatedpseudolites located at a distance of $100 \mathrm{~m}$ from the point being positioned, along azimuths: $0^{0}$, $110^{\circ}, 250^{\circ}$ some changes in the determined positions could be observed, especially in Up component of compared positions. The improvement can be seen in Figure 1.

Similar results were obtained for other directions of the PL location.Thus one can state that when 7 satellites are augmented with 3 PLs, the differences from the known position are within $\pm 7 \mathrm{~mm}$ for the $\mathrm{N}$ coordinate, $\pm 10 \mathrm{~mm}$ for $\mathrm{E}$ and $\pm 10 \mathrm{~mm}$ for the $\mathrm{Up}$ component. Let us recall that for the case of one PL, the height component was also better. The ranges of the differences decrease from $\pm 6 \mathrm{~cm}$ for the case without PL, to $-2 \mathrm{~cm}$ to $+2 \mathrm{~cm}$ for azimuth of the PL equal to $90^{\circ}$, also $-2 \mathrm{~cm}$ to $+2 \mathrm{~cm}$ for azimuth of the PL equal to $315^{\circ}$, while for the azimuths of $270^{\circ}$ and $135^{\circ}$, the approximate ranges were $-1 \mathrm{~cm}$ to $+3 \mathrm{~cm}$.

For all the studied cases, the best improvement was detected for the up component, decreasing the differences to within $\pm 1 \mathrm{~cm}$. Generally, differences of all the coordinates are within $1 \mathrm{~cm}$, but for the Up component it was obtained only after the augmentation. Without the additional distances the differences were much bigger, reaching $\pm 6 \mathrm{~cm}$. It should be emphasized that the solutions are not exactly the same as in case of classical trilateration, since the computations are performed on a basis of double differences, using real satellite phase observations as reference.

As in the previous studies, also here, the largest advantage of using pseudolites is in cases of poor satellite constellations, when the results obtained without additional distances are worse. Effect of the ground based transmitter, located at "empty" directions, is most spectacular. Such situations are presented in Figures 2 and 3. There are two obstructions used (Figs. 2a and 3a). In the first one, the satellites number $3,6,7$ are shadowed, thus 4 satellites and 3 double differenced equations remained. In the second one in spite of that also the satellite number 4 is blocked - thus only 3 satellites and 2 equations are at the disposal. Such a case is met rather often in practice when the measurements are performed near high walls, for example, in open pit mines or in urban canyons. In such cases, the PL(s) should be placed on the top of the obstruction. The PLs were located at $0^{\circ}, 270^{\circ}$ and $315^{\circ}$ and then the E and $\mathrm{N}$ coordinates are almost without any changes in comparison to these with the full 7 satellite constellation. Thus, under assumption that the pseudolite observation are accurate to about $2 \mathrm{~mm}$, it is possible to provide similar accuracy as in case of good satellite constellation. In the case presented in Figure 2, the East coordinate is a little worse and the Up component is a little better than for the reference solutions.

Also in the next case, with only 3 real satellites, where obtaining the solution without any augmentation for each epoch is impossible (only two observation equation), it can be seen that using 3 pseudolites is enough for positions to be determined, but the accuracy is a little worse than in case of original constellation. The ranges are $\pm 1.5 \mathrm{~cm}$ for $\mathrm{N}$, $\pm 3 \mathrm{~cm}$ for East and Up coordinates. Means and standard deviations for the data presented in Figures 1, 2, 3 are given in Table 1.

\section{CHANGES OF MEAN ERRORS DUE TO ADDITIONAL DISTANCES.}

Let $\mathrm{n}_{\mathrm{pl}}$ means number of PLs added. Naturally the whole adjustment for each epoch of observations may be divided into two steps: the first one where observations from only real satellites are adjusted, and 


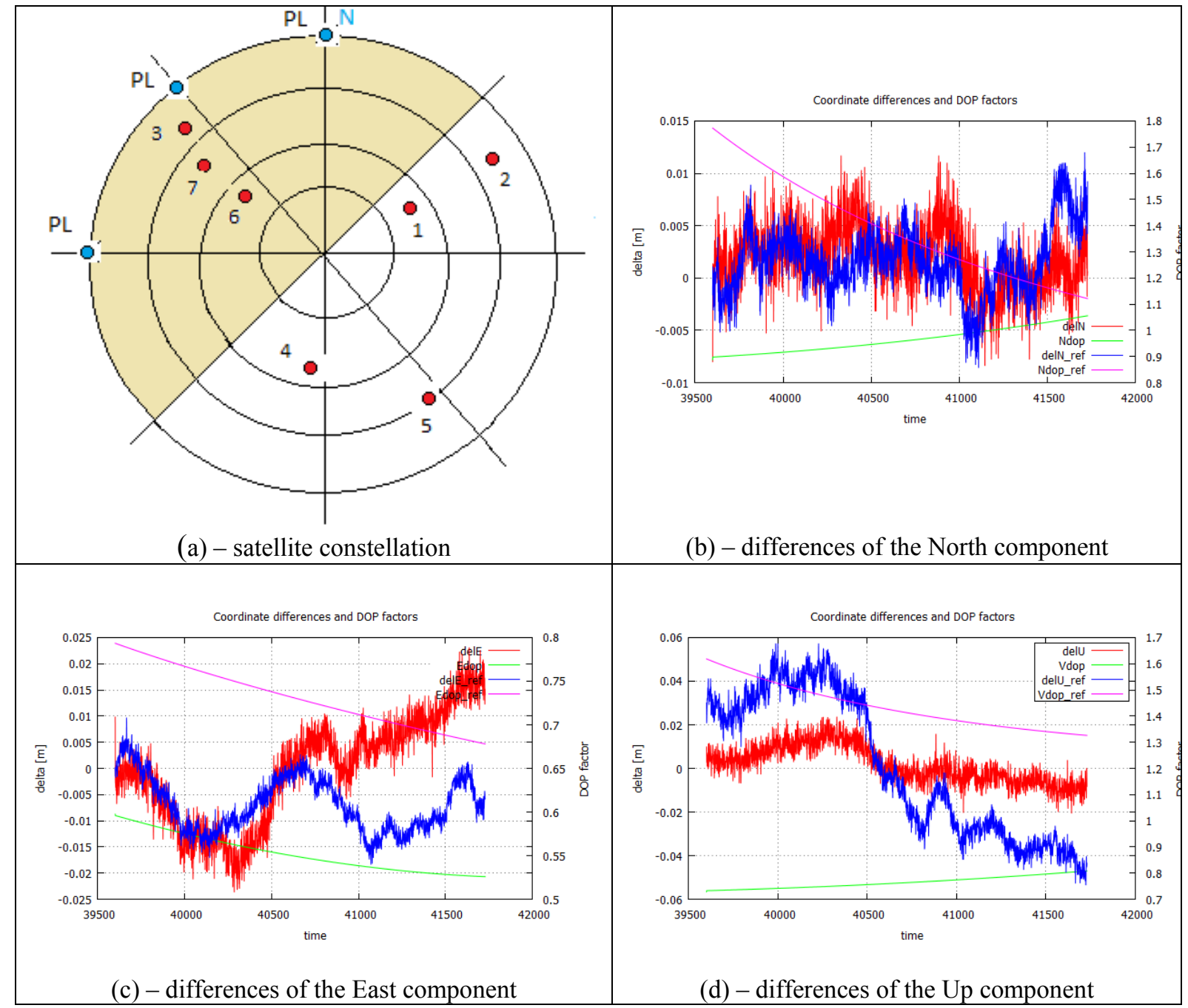

Fig. 2 Comparison of solutions with and without the PL data (simulated PL located at the azimuth of $0^{\circ}, 270^{\circ}$ and $315^{\circ}$, satellites 3, 6, 7 are shadowed); "ref" - the same as in Figure1.

Table 1 Statistics of solutions: mean differences from the value regarded as true (Mean) and their standard deviations (SD).

\begin{tabular}{lcccccc}
\hline Option & \multicolumn{2}{c}{ North } & \multicolumn{2}{c}{ East } & \multicolumn{2}{c}{ Up } \\
& Mean [m] & SD [m] & Mean [m] & SD [m] & Mean [m] & SD [m] \\
\hline 1 (ref. solution) & 0.002 & 0.003 & -0.007 & 0.005 & 0.001 & 0.032 \\
2 (data from Fig. 1) & -0.000 & 0.002 & -0.004 & 0.004 & 0.001 & 0.004 \\
3 (data from Fig. 2) & 0.002 & 0.003 & -0.000 & 0.010 & 0.002 & 0.009 \\
4 (data from Fig. 3) & 0.003 & 0.004 & 0.000 & 0.012 & -0.001 & 0.014 \\
\hline
\end{tabular}

Options. (1) All satellites, without PL; (2) All satellites, 3 PLs; (3) Satellites 3,6,7 shadowed, 3 PLs; (4) Satellites 3,4,6,7 shadowed, 3PLs (more explanations in the text)

the second one in which simulated PL-like observations are sequentially incorporated into the adjustment process. Thus we have a sequential adjustment with new observations. Let us recall that each epoch is processed independently, the only flow of information between epochs is that the parameters estimated in the previous epoch are admitted as initial coordinates in the current one. Then in the second step of the sequential adjustment the design matrix $A_{s}$, weight matrix of observations $P_{s}$ and the vector of observations and free terms $L_{s}$ have to be determined. The equations cited in (Wiśniewski, 2003) rewritten for this case will read as follows: 


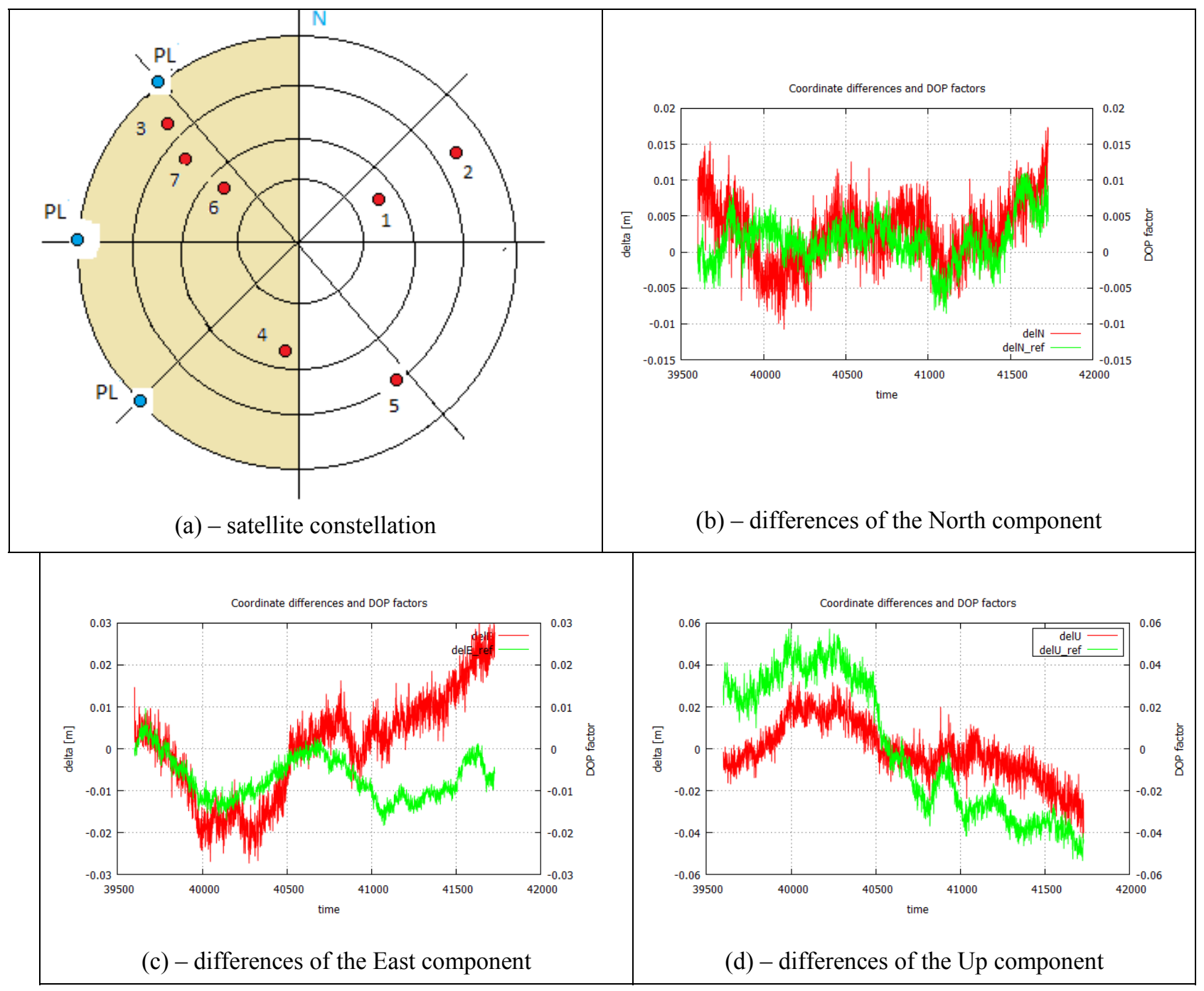

Fig. 3 Comparison of solutions with and without the PL data (simulated PL located at the azimuth of $225^{\circ}, 270^{\circ}$ and $315^{\circ}$, satellites 3, 6, 7, 4 are shadowed); "ref" - the same as in Figure 1.

$$
\begin{aligned}
& A_{S}=\left[\begin{array}{ccc}
a_{X}^{p l 1} & a_{Y}^{p l 1} & a_{Z}^{p l 1} \\
\bullet & \bullet & \bullet \\
a_{X}^{p \ln } & a_{Y}^{p \ln } & a_{Z}^{p \ln } \\
-- & -- & -- \\
1 & 0 & 0 \\
0 & 1 & 0 \\
0 & 0 & 1
\end{array}\right], L_{s}=\left[\begin{array}{c}
L^{p l 1} \\
\bullet \\
L^{p \ln } \\
-- \\
-d_{X} \\
-d_{Y} \\
-d_{Z}
\end{array}\right], \\
& P_{s}=\left[\begin{array}{cccccc}
p_{1,1}^{p l} & -1 & -1 & 0 & \bullet & 0 \\
\bullet & \bullet & \bullet & \bullet & \bullet & \bullet \\
-1 & \bullet & p_{n p l, n p l}^{p l} & 0 & \bullet & 0 \\
0 & \bullet & 0 & p_{x, 1,1} & \bullet & p_{x, 1,3} \\
\bullet & \bullet & \bullet & \bullet & \bullet & \bullet \\
0 & \bullet & 0 & p_{x, 3,1} & \bullet & p_{x, 3,3}
\end{array}\right]
\end{aligned}
$$

Thus dimensions of matrix $A_{s}$ is $\left(\mathrm{n}_{\mathrm{pl}}+3,3\right)$, of vector $L_{s}$ is $\left(\mathrm{n}_{\mathrm{pl}}+3\right)$, and these of the $P_{s}$ equal to $\left(\mathrm{n}_{\mathrm{pl}}+3, \mathrm{n}_{\mathrm{pl}}+3\right)$. First $\mathrm{n}_{\mathrm{pl}}$ raws of $A_{s}$ and $L_{s}$ are built exactly in the same way as for real satellites, similarly the first block of the $P_{s}$ matrix. Lower $(3,3)$ block of the $A_{s}$ is constituted of identity matrix, last three elements of the $L_{s}$ vector are admitted as corrections to the parameters from the first stage of adjustment, right lower $(3,3)$ block of $P_{s}$ is equal to the weight matrix of parameters estimated in the first stage of the adjustment (without PLs). Solution of the second step is given by the well known formula:

$$
d_{X}=-\left(A_{s}^{T} P_{s} A_{s}\right)^{-1} A_{s}^{T} P_{s} L_{s} \quad \text { together with its }
$$

cofactor matrix $Q_{X s}=\left(A_{S}^{T} P_{S} A_{S}\right)^{-1}$. Having the cofactor matrix $Q_{X}$ from the first step, one can compute how mean errors have changed due to addition of the new observations:

$$
\begin{aligned}
& \Delta Q_{X}=Q_{X}-Q_{X s} \quad \text { and } \quad \Delta m_{X}=m_{0} \sqrt{\left[\Delta Q_{X}\right]_{11}}, \\
& \Delta m_{Y}=m_{0} \sqrt{\left[\Delta Q_{X}\right]_{22}}, \Delta m_{Z}=m_{0} \sqrt{\left[\Delta Q_{X}\right]_{33}}
\end{aligned}
$$

These values enable assessing what is the influence of the added pseudolite-like observations onto accuracy of the results. They are plotted in Figure 5. 


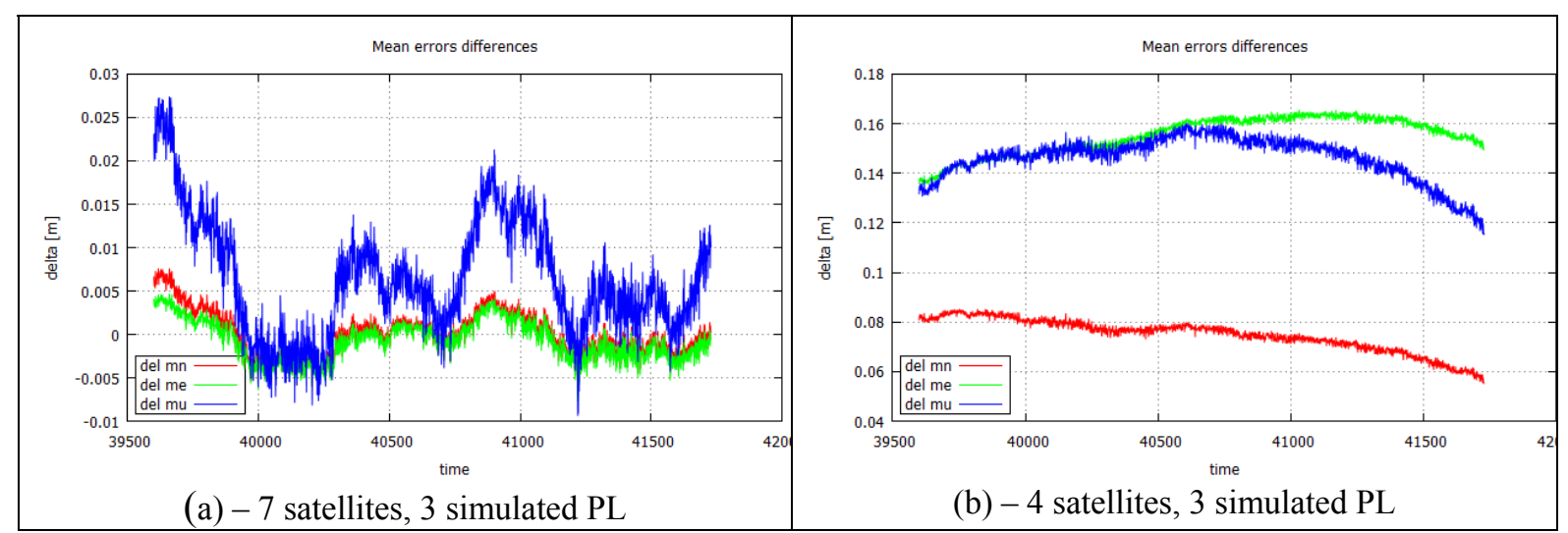

Fig. 4 Differences of mean errors for full satellite constellation and for obstruction like in Figure 2a.

Examining Figure 4 it can be stated that for the full constellation of 7 satellites, the improvements of mean errors is not very obvious, there are some epochs with even negative value of the difference, what means that formally the mean errors determined in the second step, after including additional observations, are estimated to be worse than that obtained in the first step, relying only on real satellites. But when the satellite situation becomes not so good, then the improvement of the mean errors is obvious, perhaps even a little over-estimated. Such a sequential adjustment is not possible for the second obstruction, like in Fig. 3a., thus it was only use to examine the influence of additional observations on the mean errors of unknown parameters determined.

\section{EFFECT OF ERRORS IN PSEUDOLITE OBSERVATIONS. ACCURACY PROBLEMS} MAY OCCUR WITH REAL PL OBSERVATIONS

Some errors may be rather difficult to eliminate. The most common are (Bond et al., 2007; Wang et al., 2000): the near-far problem, bigger multipath effect, different tropospheric correction and bigger linearization errors. The near-far concerns selection of an appropriate power of the signal and generally should be solved using hardware adjusting. Model given in (Grejner-Brzezińska and $\mathrm{Yi}, 2002$ ) of tropospheric corrections was checked and chosen (Rzepecka et al., 2006). It will require a verification with real PL data. The multipath can cause problems often bigger then in satellite data, since the signal travels in close vicinity of the ground. The errors tend to be constant in static applications and changing under kinematic scenario. Normally, applications like deformation monitoring, in spite of that may be computed according to kinematic algorithms, produce almost static coordinates, thus constant multipath follows. Linearization errors cause the requirement of very accurate determination of PL position as well as much more accurate approximate coordinates of the unknown points.

Taking all these into consideration, further tests have been directed to study cases where the simulated distances are burdened with larger errors then it was assumed earlier. Four scenarios have been admitted: larger random errors put on the generated distances (with standard deviation of $1 \mathrm{~cm}$ instead of previous $2 \mathrm{~mm}$ ). It was applied to PL-like signal and for comparison: to satellite No 5 distance. PL was located in the similar direction as satellite number 5 (Fig. 1a). It could be seen clearly that errors of one from three PLs observations cause larger errors in resulting positions then the same errors applied to satellite signal (Fig. 5a). In this case, all the coordinates were more contaminated with errors. Only the Up component is presented in Figure 5a. Means and standard deviations for $\mathrm{N}$ and $\mathrm{E}$ coordinates are given in Table 2. For the PL-like distance contaminated, the differences increased up to $\pm 5 \mathrm{~cm}$, while when the distance to the satellite is "spoiled" the resulting Up differences are still within $\pm 1 \mathrm{~cm}$. The next investigated case was simulation of not very accurate position of one of the PL - it was shifted by $+2 \mathrm{~cm}$ in $\mathrm{X},-1 \mathrm{~cm}$ in $\mathrm{Y}$ and $-1.8 \mathrm{~cm}$ in $\mathrm{Z}$ direction. The erroneous coordinates of PL also do cause bigger errors in results then the same errors applied to satellite coordinates (see Fig. 5b - also only the Up component is used an example). In Figure $5 \mathrm{c}$ it can be seen the influence of much worse approximate coordinates of the point being positioned (of the order of $30-40 \mathrm{~m})$. For the case without any PLs - the solutions are very good since the very first epoch, but when the PLs are used - the solution in the first epochs is shifted by almost one meter. The last studied case consisted of imposing a systematic error of $5 \mathrm{~cm}$ onto the observations to the PL and to the satellite 5 . Again, the effect of it is worse in the case of PL.

\section{CONCLUSIONS, SUMMARY, PLANS FOR FURTHER STUDIES.}

Software has been developedthat enables uniform processing of additional distance observations, expressed in cycles, together with phase GNSS observations.

Location of the PL and number of simulated PLs used can be chosen and introduced to the software. 


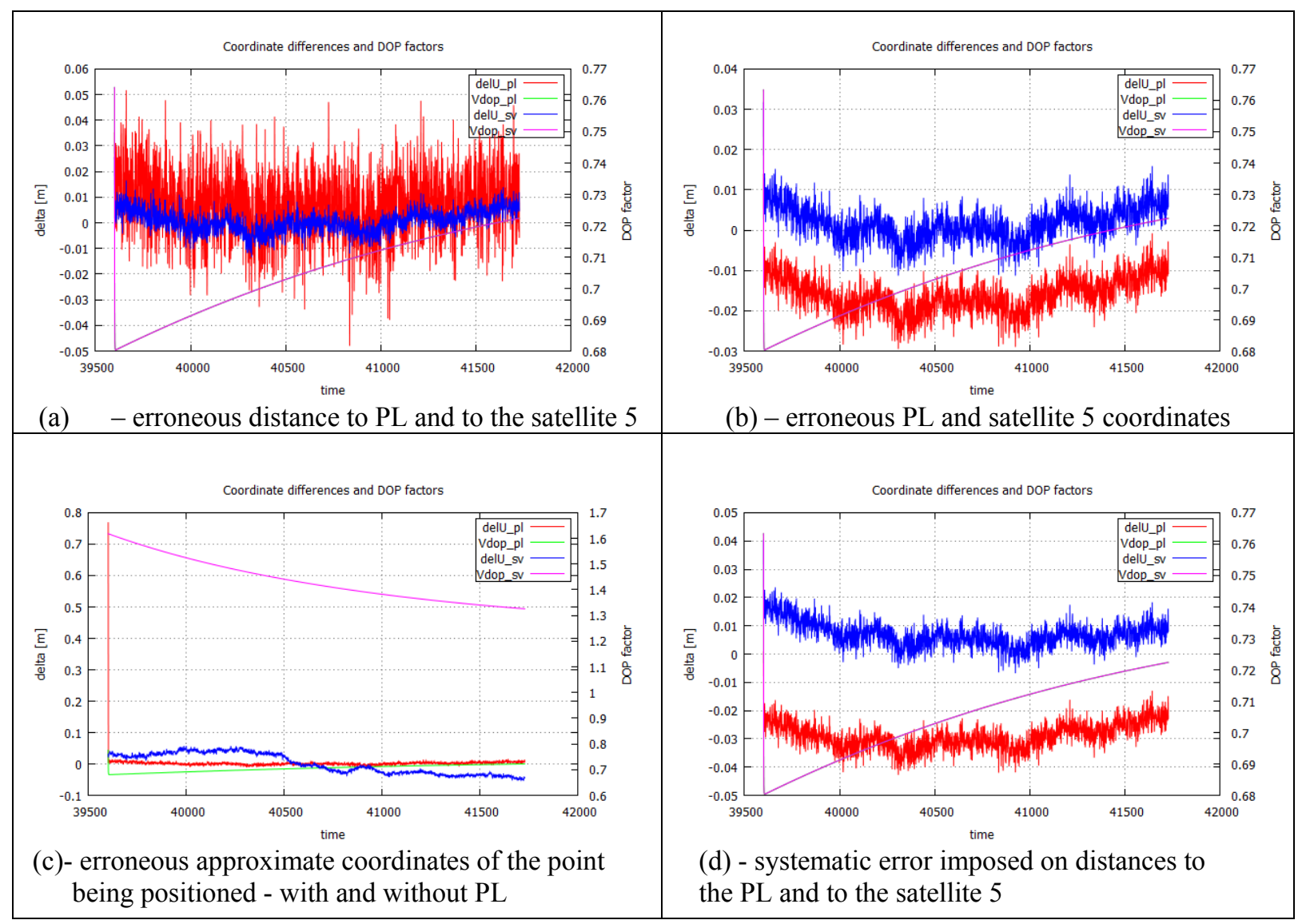

Fig. 5 Disturbances and their influence on the results, cases when original satellite observations or simulated PL observations are burdened.

Table 2 Statistics of solutions: mean differences from the value regarded as true (Mean) and their standard deviations (SD).

\begin{tabular}{lcccccc}
\hline Option & \multicolumn{2}{c}{ North } & \multicolumn{2}{c}{ East } & \multicolumn{2}{c}{ Up } \\
\cline { 2 - 7 } & Mean [m] & SD [m] & Mean [m] & SD [m] & Mean [m] & SD [m] \\
\hline 1 (data from Fig. 5a) & 0.002 & 0.005 & -0.004 & 0.004 & 0.007 & 0.013 \\
2 (data from Fig. 5a) & 0.001 & 0.003 & -0.006 & 0.005 & 0.001 & 0.004 \\
3 (data from Fig. 5b) & -0.006 & 0.003 & -0.002 & 0.004 & -0.016 & 0.004 \\
4 (data from Fig. 5b) & -0.000 & 0.002 & -0.004 & 0.004 & 0.002 & 0.004 \\
5 (data from Fig. 5c) & 0.002 & 0.003 & -0.007 & 0.005 & 0.001 & 0.032 \\
6 (data from Fig. 5c) & -0.000 & 0.004 & -0.004 & 0.004 & 0.002 & 0.017 \\
7 (data from Fig. 5d) & -0.011 & 0.003 & -0.000 & 0.004 & -0.029 & 0.005 \\
8 (data from Fig. 5d) & 0.008 & 0.002 & 0.005 & 0.005 & 0.007 & 0.032 \\
\hline
\end{tabular}

Options. (1) Distance to PL disturbed; (2) Distances to sat\# 5 disturbed; (3) PL located at incorrect coordinates; (4) Computed coordinates of sat\#5 disturbed; (5) Inaccurate approximate coordinates of the unknown station, case with all satellites, without PL; (6) Inaccurate approximate coordinates of the unknown station, case with all satellites and 3 PLs; (7) Systematic error of distance to PL; (8) Systematic error of distance to PL; (more explanations in the text; in Figs. 5a - 5d only the Up data are plotted)

The software will constitute the basis for common processing of PL together with satellite phase observations, within common adjustment procedure.

Pseudolite-like distance observations improve kinematic results, especially height component. The influence is larger for poorer satellite constellations. Use of three PLs improvesthe results in comparison with the case when only one PL is used.

Sincethe distance $\mathrm{D}$ between the reference and unknown stations in the given example was only about $500 \mathrm{~m}$, the relative errors determined for such short vectors in satellite positioning are not very impressive. For the full reference constellation, considered in this study, the relative position error may be estimated as 


$$
\frac{\Delta p}{D}=\sqrt{\frac{\sum_{i=1}^{n}\left(\Delta N_{i}^{2}+\Delta E_{i}^{2}+\Delta U_{i}^{2}\right)}{n}} / D=0.0000631 \approx 63 \mathrm{ppm}
$$

After adding 3 PLs it was changed to about $15 \mathrm{ppm}$. For the case with so severe obstructions that only 3 real satellites were remained and it was not possible to determine the position, after completing the constellation with 3 PLs, the positioning was possible and the relative error of position obtained was at the level of $45 \mathrm{ppm}$. Thus, in this case, the obtained accuracy was even better than for the original reference constellation of 7 real satellites.

The standard deviation of the noise of the simulated distances simulated was accepted to be $2 \mathrm{~mm}$. Perhaps the distances obtained from actual pseudolite measurements will havelarger random errors. Such cases were also analyzed and it has been proven that the errors imposed on PL observations cause larger errors in solutions than when they are imposed on the observations to satellites.

In these studies it was assumed that the signal from the PL is received simultaneously at two stations: reference and rover. Errors of satellite positions are cancelled in such case, but errors of PL positions are not reduced so well.

The next question that should be studied is: "could PL be replaced with a distance-meter?" The answer is not quite straightforward. There can be found pros and contras. Arguments for using PL are that signal from PL is automatically received at the phase center of the rover antenna, the same where signals from "real" satellites are received. This measurement can be double differenced and thanks to that placed into the functional positioning model together with all other satellites. The receiver clock corrections are then removed, which would not be a case with the distance not measured with the satellitelike device. Also, introducing the PL into the measurement systems, we aid technological development.

The arguments for using a classical distancemeter instead of the PL are that perhaps the distance measured would be more accurate. And that probably errors of observations using distance-meters are better recognized.There is an example of successful application of an ultra wideband distance-meter (UWB), given in literature (MacGougan and O'Keefe, 2011), it is possible that this direction of research will be also developed in the future.

\section{REFERENCES}

Bond, J., Chrzanowski, A. and Kim, D.:2007, Augmenting GPS with pseudolites for deformation monitoring in harsh environments, Proceedings of the Institute of Navigation National Technical Meeting (ION NTM), 22-24 January, San Diego, CA, USA. Available at http://www.ion.org/ and on CD-ROM.

Cellmer, S. and Rapinski, J.: 2010, Linearization problem in pseudolite surveys, Journal of Applied Geodesy, 4, 33-39 6 de Gruyter. DOI: 10.1515/JAG.2010.004
Cobb, H.S.: 1997, GPS Pseudolites: Theory, Design and Applications. PhD thesis, Stanford University.

Cosser, E.: 2004, Bridge deformation monitoring with single frequency GPS augmented by pseudolites, $\mathrm{PhD}$ thesis. University of Nottingham.

Dai, L., Zhang, J., Rizos, C., Shaowei, H. and Wang, J.: 2000, GPS and pseudolite Integration for deformation monitoring applications. Proceedings of the 10th FIG Int. Symp. on Deformation Measurements, 1-8.

Borre, K., Akos, D.M., Bertelsen, N., Rinder, P. and Jensen, S.H.: 2007, A Software-defined GPS and Galileo receiver: A Single-frequency Approach. Birkhauser.

Dach, R., Hugentobler, U., Fridez, P. and Meindl, M.: 2007, Bernese GPS Software ver. 5.0, Astronomical Institute, University of Bern.

GPS World: 2011, "Locata, a New Constellation,", September 2011, http://www.locatacorp.com/wpcontent/uploads/2011/09/GPS World 0911.pdf

Grejner-Brzezińska, D. and Yi, Y.: 2002, Experimental GPS/INS/Pseudolite System for Kinematic Positioning, Surv. Rev., 37(288), 113-126.

Hofmann-Wellenhof, B., Lichtenegger, H. and Collins, J.: 2001, Global Positioning System: Theory and Practice, Springer Wien New York.

Lemaster, E. and Rock, S.: 1999, Mars exploration using self-calibrating pseudolite arrays, 12th Int. Tech. Meeting of the Satellite Division of the U.S. Inst. of Navigation GPS ION-99, Nashville, Tennessee, 14-17 September, 1549-1558.

MacGougan, G. and O'Keefe, K.: 2009, Real Time UWB Error Estimation in a Tightly-Coupled GPS/UWB RTK Positioning System, ION ITM, Session B2.

Novariant: 2005, NovariantTerralite XPS deployment at phelps dodge morenci mine, Technical Report. Pieejams:http://www.gpsfarm.com/resources/uploads/ files/terralit, eXPS.pdf, [Access. 01 March 2008].

Pany T.: 2010, Navigation Signal Processing for GNSS Software Receivers, ARTECH HOUSEC.

Rapiński, J., Rzepecka, Z. and Cellmer, S.: 2011, Pseudolites and their applications, Proceedings of the 5-th international conference on engineering surveying, INGEO2011, Institute of Applied Geodesy Faculty of Geodesy, University of Zagreb.

Rapiński, J., Cellmer, S. and Rzepecka, Z.: 2012a, Pseudolite signal tests, Journal of Planetary Geodesy, Artificial Satellites, 47, No. 4, 147-153.

Rapiński, J., Cellmer, S. and Rzepecka, Z.: 2012b, Modified Pseudolite/GPS navigation message, The Journal of Navigation, Page 1 of 6 . The Royal Institute of Navigation. DOI: 10.1017/S0373463312000124

Rapiński, J., Cellmer, S., Rzepecka, Z. and Chrzanowski, A.: 2012, Some considerations in designing a GPS pseudolite, Journal of Planetary Geodesy, Artificial Satellites, 47, No. 1, 1-11.

DOI: $10.2478 / \mathrm{v} 10018-012-0009-7$

Rizos, C., Li, Y., Politi, N., Barnes, J. and Gambale, N.: 2011, Locata: A New Constellation for High Accuracy Outdoor and Indoor Positioning, FIG Working Week 2011, Bridging the Gap between Cultures, Marrakech, Morocco, 18-22 May 2011.

Rzepecka, Z.: 2004, Modification of Algorithms for determination of Short Kinematic Vectors from GPS Data, UWM Dissertations and Monographs, No 86, Olsztyn. 
Rzepecka, Z., Wasilewski, A., Cellmer, S. and Rapiński, J.: 2006, Integration of GPS and Pseudolites - Effect on Height Determination Accuracy, Proceedings of XI FIG Symposium on Deformation Measurements, Baden, Austria, May 2006.

Sherman, Lo: 2012, Pseudolite Alternatives for Alternate Positioning, Navigation, and Timing (APNT), Stanford University, http://www.faa.gov/about/office_org/headquarters_off ices/ato/service units/techops/navservices/gnss/library /documents/APNT/media/APNT_Pseudolite_WhitePa per_Final.pdf.

Soon, B.H.K., Poh, E.K., Barnes, J., Zhang, J., Lee, H.K. and Rizos, C.: 2003, Flight test results of precision approach and landing augmented by airport pseudolites, 16th Int. Tech. Meeting of the Satellite Division of the U.S. Institute of Navigation, Portland, Oregan, 9-12 September, 2318-2325.

Strang, G. and Borre, K.: 1997, Linear Algebra, Geodesy and GPS, Wellesley-Cambridge Press.
Tsujii, T., Harigae, M., Barnes, J., Wang, J. and Rizos, C.: 2002, Experiments of inverted pseudolite positioning for airship-based GPS augmentation system, 15th Int. Tech. Meeting of the Satellite Division of the U.S. Inst. of Navigation, Portland, Oregan, 24-27 September, 1689-1695.

Wang, J., Tsui, T., Rizos, C., Dai, L. and More, M.: 2000, Integrating GPS and Pseudolite Signals for Position and Attitude Determination: Theoretical Analysis and Experimental Results, $13^{\text {th }}$ Int. Tech. Meeting of the Satellite Division of the US Inst. of Navigation, GPS ION 2000, Salt Lake City, 2252-2262.

Wang, J.: 2002, Pseudolite Applications in positioning and navigation: Progress and problems. Journal of Global Positioning Systems, 1, No. 1, 48-56.

Wang, J.: 2007, Editorial: The Pseudolite Special Issue, Journal of Global Positioning Systems, 6, No. 2

Wiśniewski, Z.: 2003, Adjustment Calculus in Geodesy (in Polish), University of Warmia and Mazury, Olsztyn, Poland. 